\title{
Interval-based and Fuzzy Set-based Approaches to Modeling of Fuzzy Inference Systems with the Local Monotonicity Property
}

\author{
${ }^{1}$ Chin Ying Teh, ${ }^{1 *}$ Kai Meng Tay, ${ }^{2}$ Chee Peng Lim \\ ${ }^{1}$ Faculty of Engineering, Universiti Malaysia Sarawak, Kota Samarahan, Sarawak, Malaysia. \\ ${ }^{2}$ Centre for Intelligent Systems Research, Deakin University, Australia \\ *kmtay@,feng.unimas.my
}

\begin{abstract}
Even though the importance of the local monotonicity property for function approximation problems is well established, there are relative few investigations addressing issues related to the fulfillment of the local monotonicity property in Fuzzy Inference System (FIS) modeling. We have previously conducted a preliminary study on the local monotonicity property of FIS models, with the assumption that the extrema point(s) (i.e., the maximum and/or minimum point(s)) is either known precisely or totally unknown. However, in some practical situations, the extrema point(s) can be known imprecisely (as an interval or a fuzzy set). In this paper, the imprecise information is exploited to construct an FIS model that fulfills the local monotonicity property. A procedure to estimate the extrema point(s) of a function is devised. Applicability of the findings to a datadriven modeling problem is further demonstrated.
\end{abstract}

Keywords-Fuzzy inference system; local monotonicity; monotonicity test; interval approach; fuzzy set approach; datadriven modeling

\section{INTRODUCTION}

A search in the literature reveals that recent studies on Fuzzy Inference System (FIS) modeling are mainly focused on issues related to the fulfillment of theoretical properties, i.e., monotonicity [1-2], robustness [3-4], continuity [5], and local monotonicity [6-7]. The main emphasis of this paper is on the local monotonicity property of a single-input function. Consider a single input function, $y=f(x)$, that fulfills the condition of local monotonicity between its output, $y$, with respect to $x$, within a universe of discourse. If an FIS model fulfills the local monotonicity property between $y$ and $x$ within the upper and lower bounds of $x$ (denoted as $\bar{x}$ and $\underline{x}$, respectively), where the limit between the upper and lower bounds is a subset of the universe discourse, $y$ monotonically increases or decreases as $x$ increases, i.e., $f\left(x_{1}\right) \leq f\left(x_{2}\right)$ or $f\left(x_{1}\right) \geq f\left(x_{2}\right)$ respectively for $x_{1}<x_{2}$, within the limit.

The concept of the local monotonicity property and the importance of this line of study have been highlighted in the recent studies on signal processing [8-10]. Indeed, a number of local monotonicity preserving techniques, which include local monotonicity regression [8], multidimensional local monotonicity [9], and local monotonicity diffusion [10], have been proposed for a variety of signal processing applications. However, a search in the literature reveals that the study on the local monotonicity property of FIS models is still new.

In our previous studies [6-7], some preliminary investigations on the local monotonicity property of FIS models have been reported. In [6], the local monotonicity property has been exploited as an additional piece of qualitative information for FIS modeling. The extrema point(s) (maximum and/or minimum point (s)) of an FIS model is assumed to be known precisely [6]. In [7], the use of an optimization-based similarity reasoning scheme to problems that involve the local monotonicity property has been demonstrated. We have developed a procedure to estimate the trend (static, increasing, or decreasing) of a function, and to record the number of changes in the respective trend [7]. Such an approach is useful when the extrema point(s) is unknown. In this paper, we further extend our previous studies in [6-7] to handle situations whereby the extrema point(s) of a function is known imprecisely, instead of precisely known or totally unknown.

The aim of this paper is to study a general approach to construct an FIS model that is able to preserve the local monotonicity property. Instead of assuming the extrema point(s) (i.e., the maximum and/or minimum point(s)) to be known precisely or unknown totally, it is assumed that the extrema point(s) is known imprecisely. This imprecise information is exploited as additional qualitative information for FIS modeling. In this paper, two new approaches (i.e., interval-based and fuzzy set-based) to model the extrema point(s) of a function, is firstly proposed. It is worth mentioning that the interval-based approach is a special case of the fuzzy set-based approach. Such modeling is useful because real-world data are often imprecise, owing to a number of reasons, e.g. missing data, instrument noise, measurement and approximation error in computational models.

A procedure to estimate the extrema point(s) of a function is further devised. With the devised procedure, the estimated extrema point(s) is obtained. The degree or membership of the estimated extrema point(s) to satisfy an interval-based and/or fuzzy set-based model is further obtained. Such information allows an FIS model to be constructed with an optimization-based FIS modeling 